\title{
Características clínicas, epidemiológicas y microbiológicas de una cohorte de pacientes con tuberculosis pulmonar en Cali, Colombia
}

\author{
Christian Mauricio Rojas ${ }^{1,2}$, Sonia Lorena Villegas ${ }^{1,2}$, Hildegard María Piñeros ${ }^{1,2}$, \\ Ennid Margarita Chamorro ${ }^{1,2}$, Carlos Eduardo Durán ${ }^{1,2}$, Edna Lorena Hernández ${ }^{1,2}$, \\ Robinson Pacheco ${ }^{1,2}$, Beatriz Eugenia Ferro ${ }^{1,2}$ \\ 1 Área de Tuberculosis, Centro Internacional de Entrenamiento e Investigaciones Médicas (CIDEIM), Cali, \\ Colombia \\ 2 Centro Colombiano de Investigación en Tuberculosis (CCITB), Medellín, Colombia
}

Introducción. El tratamiento acortado estrictamente supervisado hace parte de una estrategia multifactorial adoptada por la Organización Mundial de la Salud para controlar la tuberculosis y en Colombia alcanza una cobertura de $70 \%$.

Objetivo. Establecer las características clínicas, epidemiológicas y microbiológicas y el desenlace de la terapia antituberculosa, en una cohorte de pacientes nuevos con diagnóstico de tuberculosis pulmonar con baciloscopia positiva en Cali, Colombia.

Materiales y métodos. Se llevó a cabo un estudio descriptivo, anidado en un ensayo clínico de multicéntrico, en el que se incluyeron 106 pacientes con tuberculosis pulmonar con baciloscopia positiva entre abril de 2005 y junio de 2006, a través de instituciones de salud de la red pública de Cali que administraron el tratamiento antituberculoso.

Se practicó baciloscopia seriada, cultivo para micobacterias, prueba de sensibilidad a medicamentos antituberculosos de primera línea, radiografía de tórax postero-anterior y lateral y prueba ELISA para VIH. Se recolectó información clínica y epidemiológica, y se hizo seguimiento por 30 meses, haciendo uso de incentivos de transporte y alimentación.

Resultados. La mayoría de los pacientes fueron hombres jóvenes, con diagnóstico hecho más de nueve semanas después del inicio de los síntomas y con baciloscopia muy positiva $(2+03+)$. La resistencia a cualquier medicamento fue de $7,5 \%$ y la resistencia inicial a los medicamentos de primera línea fue de 1,9\%. La incidencia de efectos secundarios asociados al tratamiento fue de 8,5\%. La infección concomitante con VIH fue de 5,7\%. El 86,8\% de los pacientes completó la terapia con diagnóstico de curación.

Conclusiones. El período tan amplio comprendido entre el inicio de los síntomas y el diagnóstico de la enfermedad, se ha identificado previamente como factor de riesgo para el abandono de terapia. Las características epidemiológicas son similares a las descritas para los pacientes con tuberculosis pulmonar. Los hallazgos radiográficos indicativos de tuberculosis son diversos y no corresponden en su mayoría a cavernas. La infección concomitante con VIH fue identificada como parte del abordaje de la enfermedad por micobacterias en todos los casos.

Palabras clave: tuberculosis pulmonar, resultado del tratamiento, curación, Colombia.

Clinical, epidemiological and microbiological characteristics of a cohort of pulmonary tuberculosis patients in Cali, Colombia

Introduction. The World Health Organization recommended strategy for global tuberculosis control is a short-course, clinically administered treatment, This approach has approximately $70 \%$ coverage in Colombia.

Objective. The clinical, epidemiological and microbiological characteristics along with drug therapy outcomes were described in newly diagnosed, pulmonary tuberculosis patients.

Materials and methods. This was a descriptive study, conducted as part of a multicenter clinical trial of tuberculosis treatment. A cohort of 106 patients with pulmonary tuberculosis were recruited from several public health facilities in Cali between April 2005 and June 2006. Sputum smear microscopy, culture, drug susceptibility tests to first-line anti-tuberculosis drugs, chest X-ray and HIV-ELISA were performed. Clinical and epidemiological information was collected for each participant. Treatment was administered by the local tuberculosis health facility. Food and transportation incentives were provided during a 30 month follow-up period.

Results. The majority of patients were young males with a diagnostic delay longer than 9 weeks and a high sputum smear grade (2+ or $3+)$. The initial drug resistance was $7.5 \%$ for single drug treatment and $1.9 \%$ for multidrug treatments. The incidence of adverse events associated with treatment was $8.5 \%$. 
HIV co-infection was present in $5.7 \%$ of the cases. Eighty-six percent of the patients completed the treatment and were considered cured. The radiographic presentation varied within a broad range and differed from the classic progression to cavity formation.

Conclusion. Delay in tuberculosis diagnosis was identified as a risk factor for treatment compliance failure. The study population had similar baseline epidemiologic characteristics to those described in other cohort studies.

Key words: Tuberculosis, pulmonary; treatment outcome, cure, Colombia.

La tuberculosis ha afectado a la humanidad por más de 7.000 años y es actualmente la segunda causa de mortalidad debida a una enfermedad infecciosa a nivel mundial, al ocasionar la muerte de 1,77 millones de personas cada año (1). En el año 2007 hubo 9,27 millones de casos nuevos de tuberculosis; de éstos, 4,1 millones de casos tuvieron baciloscopia positiva ( $44 \%$ del total) y 1,37 millones estaban infectados simultáneamente con $\mathrm{VIH}(8 \%$ del total). El $95 \%$ de los casos de tuberculosis y el $98 \%$ de los fallecimientos por esta causa suceden en países de bajo y mediano ingreso, y la población económicamente activa (15 a 54 años) es la más afectada, con $75 \%$ de los casos (1).

El tratamiento acortado estrictamente supervisado -Directly Observed Treatment, Short-course, DOTShace parte de la estrategia "Alto a la TB", establecida como política por la Asamblea Mundial de la Salud y promovida por la Organización Mundial de la Salud (OMS) para el control de la enfermedad, basada en: 1) el compromiso gubernamental, 2) la detección oportuna mediante baciloscopia en sintomáticos respiratorios, 3) el tratamiento acortado estandarizado para, al menos, todos los casos con baciloscopia positiva, 4) el acceso a los medicamentos y 5) el sistema de seguimiento, supervisión y evaluación de pacientes. Desde el año 2006, la OMS busca la expansión de un DOTS de calidad y mejorado, hace énfasis en la protección a las poblaciones vulnerables, la identificación y abordaje de la infección simultánea tuberculosis/VIH y la tuberculosis resistente a los medicamentos de primera línea, entre otros (2).

La OMS ha establecido una meta de cobertura de DOTS con un mínimo de $70 \%$ y Colombia se encuentra en dicho porcentaje de cobertura (1). El número de casos de tuberculosis reportados en el

\footnotetext{
Correspondencia:

Christian Mauricio Rojas, Bridgeport Hospital, Internal Medicine Program, Yale University, 267 Grant Street, Bridgeport, CT 06610, USA

Teléfono: (203) 415 6143; fax: (203) 3844294

qtextian@hotmail.com
}

Recibido: 14/08/09; aceptado:10/06/10
2007 fue de 10.950 (24 por 100.000 habitantes), de los cuales, cerca de $85 \%$ fueron pulmonares (1). En Cali, el Programa de Control de la Tuberculosis informó en el 2007, 1.019 casos de todas las formas de la enfermedad, para una incidencia de 41,13 por 100.000 habitantes, lo que se encuentra por encima de la media nacional. En el mismo año, se presentaron 96 casos mortales $(9,4 \%)$ y la infección simultánea con VIH se detectó en 94 casos $(9,2 \%)$, lo cual superó las cifras nacionales para ese año $(1,3)$.

El conocimiento de las características de las cohortes de pacientes con tuberculosis pulmonar es fundamental para el fortalecimiento de la estrategia DOTS a nivel regional, con el objetivo de lograr mayores porcentajes de curación y disminuir el de abandono. Sin embargo, es poca la información reciente publicada sobre la tuberculosis en Cali. Por tal razón, este estudio se propuso describir las características clínicas, epidemiológicas y microbiológicas de la tuberculosis en la ciudad y el desenlace de la terapia antituberculosa después de un seguimiento estricto. Los hallazgos reportados pueden ser de utilidad para los trabajadores de la salud y el Programa de Control de la Tuberculosis que se enfrentan a la detección, diagnóstico y manejo de esta enfermedad.

\section{Materiales y métodos}

\section{Población y diseño de estudio}

Este es un estudio descriptivo de una cohorte de 106 pacientes en Cali, que participó en un ensayo clínico multicéntrico que evaluó dos presentaciones diferentes del tratamiento para tuberculosis pulmonar (Estudio C). No se incluyen los hallazgos diferenciales según los esquemas terapéuticos evaluados en el ensayo clínico.

Para la conducción del estudio, los pacientes fueron seleccionados entre abril de 2005 y junio de 2006, a través de las ESE (Empresa Social del Estado) Norte, Centro, Oriente y Suroriente de Cali. Se utilizaron los siguientes criterios de inclusión:

a) tener, al menos, dos baciloscopias de esputo positivas; 
b) no haber recibido previamente terapia antituberculosa;

c) ser mayor de 18 años;

d) tener la intención de residir en un domicilio permanente, $y$

e) autorizar su participación en el estudio y la práctica de una prueba de $\mathrm{VIH}$ mediante consentimiento informado.

Los criterios de exclusión fueron:

a) estado agonizante;

b) meningitis tuberculosa;

c) diabetes de tipo 1, enfermedades del hígado o el riñón, alteraciones sanguíneas, neuritis periférica;

d) embarazo o período de lactancia;

e) enfermedad psiquiátrica, y

f) contraindicación a algún fármaco en el esquema del estudio.

Se incluyeron los pacientes con enfermedad pulmonar crónica (EPOC), tabaquismo, alcoholismo, abuso de psicotóxicos, diabetes de tipo 2 y desnutrición.

\section{Consideraciones éticas}

Para la realización de este estudio se contó con la aprobación del Comité Institucional de Ética de Investigaciones en Humanos del Centro Internacional de Entrenamiento e Investigaciones Médicas (CIDEIM). Igualmente, se obtuvo la aprobación de las instituciones participantes y de la Secretaría de Salud Pública Municipal.

\section{Recolección de información}

Luego de aceptar voluntariamente participar en el estudio, todos los pacientes fueron valorados por personal médico del CIDEIM, que diligenció un cuestionario estructurado para la recolección de datos e hizo pruebas de tamización para diabetes y prueba de embarazo. A los pacientes que satisfacían los criterios de inclusión se les practicó radiografía de tórax en proyecciones posteroanterior y lateral, prueba ELISA para $\mathrm{VIH}$, se les tomaron muestras de esputo para baciloscopia y cultivo de micobacterias, y se les hizo prueba de sensibilidad a medicamentos antituberculosos de primera línea.

El tratamiento se administró a todos los pacientes mediante la estrategia DOTS, por instituciones participantes del estudio que, a su vez, ejecutan usualmente las actividades del Programa de Control de la Tuberculosis en Cali.

El tratamiento incluyó isoniacida, rifampicina, etambutol y pirazinamida, según dosis recomendadas por las guías de la OMS. El desenlace de la terapia se definió de acuerdo con las categorías del programa: fracaso (baciloscopia positiva al quinto mes de tratamiento), curación (baciloscopia negativa al finalizar el tratamiento), tratamiento terminado (dosis totales cumplidas, pero sin baciloscopia al final del tratamiento), recaída (baciloscopia positiva después de haberse establecido la curación), abandono (falta de cumplimiento de la terapia mayor de 30 días continuos), transferido (remisión a otra institución o programa para continuar la terapia).

Esta cohorte se siguió durante los seis meses de tratamiento y por 24 meses adicionales, para un total de 30 meses, en los cuales se documentó la evolución clínica y microbiológica por medio de visitas de seguimiento que cumplían los pacientes en el CIDEIM, con apoyo de seguimiento telefónico e incentivos de transporte. Asimismo, durante el seguimiento se entregaron incentivos alimenticios para el paciente y su familia.

Se registró la presentación de efectos secundarios a la medicación, cuando los pacientes lo manifestaron espontáneamente. El efecto secundario se clasificó como leve, cuando no comprometía el estado funcional; moderado, si comprometía el estado funcional y requería tratamiento, y grave, cuando se presentaba un mayor compromiso funcional, requería atención hospitalaria o significaba una amenaza para la vida. Se tomó una muestra de esputo para baciloscopia y cultivo en los meses 2 , $3,5,6,8,10,12,15,18,24$ у 30.

La baciloscopia directa se coloreó por el método de Ziehl-Neelsen y, para el cultivo, se descontaminó la muestra con $\mathrm{NaOH}$ al $4 \%$ y se sembró directamente en medio Ogawa-Kudoh (4). La identificación de Mycobacterium tuberculosis en los cultivos positivos se hizo mediante pruebas bioquímicas (5). Las pruebas de sensibilidad a los medicamentos antituberculosos de primera línea se practicaron por el método de proporciones múltiples en agar Middlebrook $7 \mathrm{H} 10$ (5), utilizando los siguientes antibióticos y concentraciones: $1,0 \mu \mathrm{g} / \mathrm{ml}$ de isoniacida, $1,0 \mu \mathrm{g} / \mathrm{ml}$ de rifampicina, $5,0 \mu \mathrm{g} / \mathrm{ml}$ de etambutol y 2,0 $\mu \mathrm{g} / \mathrm{ml}$ de estreptomicina. Se evaluó indirectamente la sensibilidad a la pirazinamida, mediante la prueba de la pirazinamidasa. 
Se clasificó como tuberculosis resistente a los medicamentos de primera línea a todo aislamiento que presentara resistencia, al menos, a isoniacida y rifampicina, interpretada como resistencia primaria o inicial debido a que todos los pacientes fueron nuevos (tuberculosis por primera vez). Se contó con control de calidad externo indirecto para la identificación de $M$. tuberculosis y las pruebas de sensibilidad, el cual fue realizado por la Unidad de Micobacteriología del Instituto de Medicina Tropical de Amberes, Bélgica, a través de la Unión Internacional Contra la Tuberculosis y Enfermedades Respiratorias.

\section{Análisis de datos}

Se elaboró una base de datos en el programa Microsoft Access 2003® y los datos se analizaron mediante el programa SPSS versión 7,5, utilizando $\chi^{2}$ para las pruebas de asociación entre variables. Un valor de $p$ menor de 0,05 se consideró como significativo en todos los análisis estadísticos.

\section{Resultados}

\section{Hallazgos iniciales}

Se hizo seguimiento a 106 pacientes. El análisis de la información demográfica mostró que la población de estudio estuvo compuesta, principalmente, por adultos jóvenes (18 a 34 años), con un ligero predominio del sexo masculino (cuadro 1). La tos por más de dos semanas y la expectoración sin hemoptisis constituyeron los síntomas cardinales en la cohorte. Los síntomas constitucionales se manifestaron en cerca de $70 \%$ de la población de estudio (cuadro 2).

El tiempo entre el inicio de la sintomatología y la confirmación diagnóstica fue mayor de nueve semanas en más de la mitad de los casos.

En el examen microbiológico inicial, $80 \%$ de los pacientes presentó baciloscopia de cuantificación $2+$ y 3+, y en 105 pacientes se aisló $M$. tuberculosis de la muestra de esputo. En un paciente que presentó infección concomitante $\mathrm{VIH} /$ tuberculosis se encontró una micobacteria no tuberculosa como la causa de la infección pulmonar, identificada como Mycobacterium kansasii en un laboratorio de referencia, por lo que se excluyó del análisis.

Según el examen radiográfico previo al inicio de la terapia antituberculosa, practicado a 104 de 106 pacientes, la localización de los hallazgos incluyó alguno de los lóbulos superiores en $72 \%$ de los casos, con infiltrados intersticiales retículonodulares como hallazgo principal. Se encontraron
Cuadro 1. Características sociodemográficas de 106 pacientes con tuberculosis pulmonar con baciloscopia positiva en Cali, Colombia.

\begin{tabular}{lrr}
\hline $\begin{array}{l}\text { Características } \\
\text { sociodemográficas }\end{array}$ & Número & Porcentaje \\
\hline Número de casos & 106 & \\
Sexo & & \\
$\quad$ Masculino & 55 & 51,9 \\
$\quad$ Femenino & 51 & 48,1 \\
Mediana de la edad (años) & & \\
(rango, 18 a 95) & 33,0 & \\
Distribución de la edad (años) & & \\
$\quad 224$ & 28 & 26,4 \\
25 a 34 & 30 & 28,3 \\
35 a 44 & 16 & 15,1 \\
45 a 54 & 17 & 16,0 \\
55 a 64 & 9 & 8,5 \\
$\geq 65$ & 6 & 5,7 \\
Estrato socioeconómico & & \\
1 & 27 & 25,5 \\
2 & 59 & 55,7 \\
3 & 11 & 10,4 \\
4 & 2 & 1,6 \\
Sin dato & 7 & 6,6 \\
Régimen de salud & & \\
Contributivo & 22 & 20,8 \\
Subsidiado & 35 & 33,0 \\
No asegurado & 47 & 44,4 \\
Sin dato & 2 & 1,9 \\
\hline
\end{tabular}

imágenes indicativas de cavernas en 35 de 104 casos y atelectasia lobar en 8 casos, 7 de ellas descritas en el lóbulo superior derecho y 1 en el lóbulo inferior derecho. Hubo 5 de 104 que presentaron consolidación lobar: 3 en el lóbulo superior derecho, 1 en el lóbulo superior izquierdo y 1 en el lóbulo medio derecho. Se describió derrame pleural en 2 de 104. Asimismo, se encontró neumotórax derecho de $15 \%$ en un solo caso y lesión fibronodular con reacción de pleura apical y lesiones fibrosas que involucraban ambos lóbulos superiores, en otro caso. En un caso con infección concomitante $\mathrm{VIH} /$ tuberculosis, la radiografía de tórax fue normal. Se encontró asociación entre la presencia de enfermedad pulmonar bilateral y la edad igual o mayor de 55 años y, también, con la ausencia de cicatriz por BCG $(p<0,05)$.

La infección concomitante con VIH estuvo presente en 6 casos, en 5 de ellos se inició con tuberculosis pulmonar sin otras infecciones oportunistas previas. El sexto caso correspondió a la infección por M. kansasii. Tres de los casos tuvieron acceso a terapia antirretroviral.

Entre las principales enfermedades concomitantes en el momento del diagnóstico de tuberculosis pulmonar, se encontraron: diabetes mellitus de 
Cuadro 2. Características clínicas y bacteriológicas de la tuberculosis pulmonar en 106 pacientes con baciloscopia positiva en Cali, Colombia.

\begin{tabular}{|c|c|c|}
\hline Características clínicas & Número & Porcentaje \\
\hline Número de casos & 106 & \\
\hline \multicolumn{3}{|l|}{ Sintomatología al momento del diagnóstico } \\
\hline Expectoración & 93 & 87,7 \\
\hline Tos durante más de 15 días & 92 & 86,8 \\
\hline Pérdida del apetito & 73 & 68,9 \\
\hline Pérdida de peso & 69 & 65,1 \\
\hline Fiebre & 69 & 65,1 \\
\hline Sudoración nocturna & 62 & 58,5 \\
\hline Disnea & 28 & 26,4 \\
\hline Hemoptisis & 13 & 12,3 \\
\hline \multicolumn{3}{|l|}{ Mediana del retraso diagnóstico total en semanas } \\
\hline (rango, 1,4 a 63,1 ) & 9,4 & \\
\hline \multicolumn{3}{|l|}{ Mediana del índice de masa corporal $\left(\mathrm{kg} / \mathrm{m}^{2}\right)$} \\
\hline (rango, 14,4 a 27,0 ) & 19,5 & \\
\hline Bajo peso (índice de masa corporal $<18,5$ ) & 48 & 45,3 \\
\hline \multicolumn{3}{|l|}{ Resultados de exámenes practicados } \\
\hline \multicolumn{3}{|l|}{ Radiografía de tórax } \\
\hline Enfermedad con cavidades & 35 & 33,6 \\
\hline Enfermedad bilateral & 56 & 53,8 \\
\hline \multicolumn{3}{|l|}{ Baciloscopia } \\
\hline 10 a 99 BAAR en 100 campos (1+) & 20 & 18,9 \\
\hline 1 a 10 BAAR por campo $(2+)$ & 22 & 20,8 \\
\hline$>10$ BAAR por campo $(3+)$ & 64 & 60,4 \\
\hline Cicatriz por BCG & 52 & 49,1 \\
\hline Contacto previo con paciente tuberculoso & 16 & 15,1 \\
\hline \multicolumn{3}{|l|}{ Enfermedades concomitantes } \\
\hline Coinfección VIH & 6 & 5,7 \\
\hline Diabetes de tipo 2 & 4 & 3,8 \\
\hline EPOC & 4 & 3,8 \\
\hline Tabaquismo & 23 & 21,7 \\
\hline Consumo crónico de alcohol & 12 & 11,3 \\
\hline
\end{tabular}

BAAR: bacilos ácido-alcohol resistentes; BCG: bacilo de Calmette y Guérin; VIH: virus de la inmunodeficiencia humana; EPOC: enfermedad pulmonar obstructiva crónica

tipo 2, EPOC, tabaquismo y consumo crónico de alcohol (cuadro 2).

Las pruebas de sensibilidad a los medicamentos de primera línea, mostraron resistencia inicial o primaria a alguno de los medicamentos evaluados en $7,6 \%$ de los aislamientos de $M$. tuberculosis, mientras que la resistencia inicial o primaria fue de $1,9 \%$ (cuadro 3). No se encontró asociación entre la resistencia antimicrobiana y las características demográficas, las clínicas y la presentación de efectos adversos a la medicación.

\section{Desenlace de la terapia antituberculosa}

De los 105 pacientes incluidos, 92 (87,6\%) completaron la terapia con diagnóstico de curación. Durante la terapia fallecieron tres pacientes $(2,8 \%)$, uno de ellos por tuberculosis, que se trataba de un caso de resistencia a los medicamentos de primera línea, y los dos restantes por causas no asociadas con la enfermedad (figura 1). Nueve pacientes $(8,5 \%)$ abandonaron la terapia antituberculosa
Cuadro 3. Resistencia primaria a medicamentos antituberculosos de primera línea en 105 pacientes con tuberculosis pulmonar en Cali, Colombia.

\begin{tabular}{lcc}
\hline & $\begin{array}{c}\text { Número de } \\
\text { casos† }\end{array}$ & Porcentaje \\
\hline Tipo de resistencia & 105 & \\
A cualquier medicamento & 8 & 7,6 \\
Resistencia a la isoniacida & 4 & 3,8 \\
Resistencia a la estreptomicina & 1 & 0,9 \\
Isoniacida más estreptomicina & 1 & 0,9 \\
Resistencia a los medicamentos & 2 & 1,9 \\
de primera línea & & \\
\hline
\end{tabular}

† El caso de Mycobacterium kansasii se excluyó del análisis de resistencia.

y no reingresaron al Programa de Control de la Tuberculosis o al estudio. El 80,6\% (79/98) de los pacientes presentó cultivo negativo al segundo mes de seguimiento.

Una vez obtenidos los resultados de las pruebas de sensibilidad a medicamentos antituberculosos, 


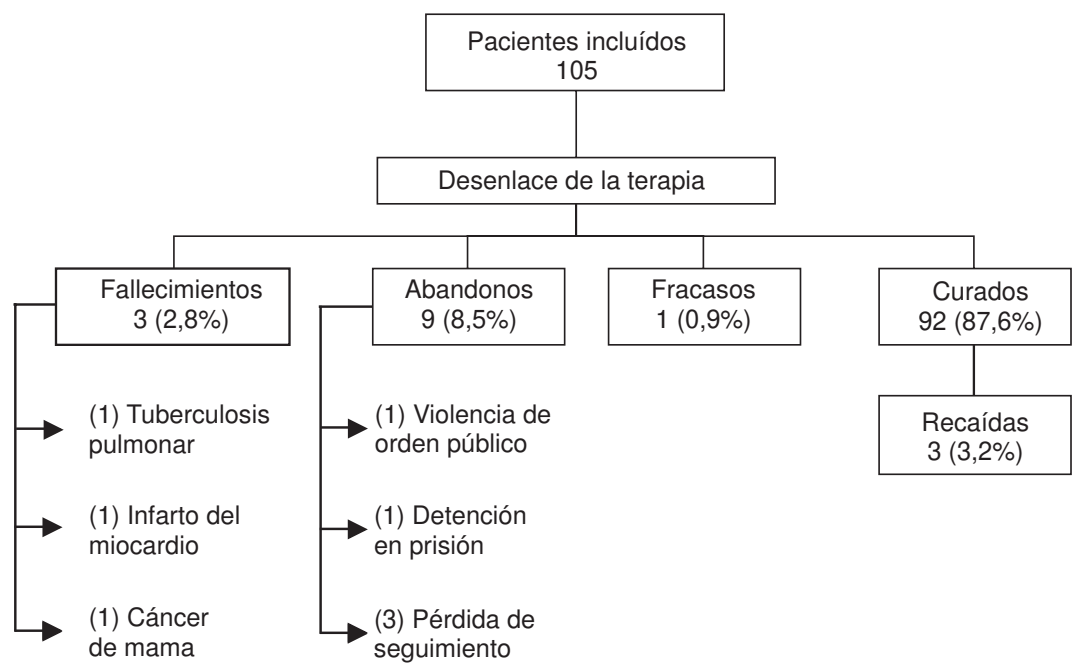

Figura 1. Perfil del estudio, cohorte de 105 pacientes con tuberculosis pulmonar en Cali, Colombia.

se detectaron cinco casos de resistencia a un medicamento: cuatro a isoniacida y uno a estreptomicina. Estos pacientes finalizaron la terapia con diagnóstico de curación y sin recaída por tuberculosis pulmonar durante el seguimiento (cuadro 3).

Se detectó un caso de resistencia inicial o primaria combinada, a isoniacida y estreptomicina, en un paciente que había requerido protocolo de reintroducción de medicamentos debido a hipersensibilidad a la pirazinamida. Este caso presentó baciloscopia y cultivo positivos al sexto mes de tratamiento, y se convirtió en un fracaso terapéutico. Una nueva prueba de sensibilidad evidenció tuberculosis resistente a los medicamentos de primera línea, por lo cual fue necesario el tratamiento con medicamentos de segunda línea e intervenciones quirúrgicas posteriores, sin lograrse la curación. Tuvo dificultad en el acceso al tratamiento y graves problemas en su cumplimiento.

Uno de los dos pacientes de tuberculosis resistente a los medicamentos de primera línea inicial o primaria falleció al mes siguiente de la detección de dicha resistencia, sin tener acceso a medicamentos de segunda línea y en condición de desnutrición avanzada. El segundo caso interrumpió el esquema terapéutico con medicamentos de segunda línea al cuarto mes de haberse iniciado, al perder la afiliación al régimen de seguridad social en salud. El paciente se tornó asintomático por 24 meses, tiempo durante el cual los cultivos de seguimiento fueron negativos. No obstante, presentó nuevamente un cuadro sugestivo de tuberculosis y la prueba de sensibilidad demostró una tuberculosis resistente a los medicamentos de primera línea; requirió manejo quirúrgico posterior, sin lograr completar el esquema terapéutico por 24 meses debido a problemas de cumplimiento.

Después de finalizada la terapia antituberculosa, 92 pacientes permanecieron en seguimiento. En los primeros dos a cuatro meses desde la finalización del tratamiento, se encontraron $3(3,2 \%)$ casos de recaída, diagnosticados por cultivo en pacientes con baciloscopias positivas al ingreso del estudio de $2+$ y $3+$. No se encontró adquisición de resistencia según prueba de sensibilidad realizada en dichas recaídas. Solamente la presencia de hemoptisis al momento del diagnóstico inicial estuvo asociada

Cuadro 4. Efectos adversos asociados a terapia antituberculosa en 105 pacientes con tuberculosis pulmonar en Cali, Colombia.

\begin{tabular}{lcc}
\hline Característica & Número & Porcentaje \\
\hline Número de casos $†$ & 105 & \\
Número de efectos adversos observados & 9 & 8,5 \\
Seriedad del efecto secundario & & \\
$\quad$ Leve & 3 & 2,8 \\
Moderado & 4 & 3,8 \\
$\quad$ Grave & 2 & 1,9 \\
Tipo de efecto secundario & 1 & 0,9 \\
Síndrome pseudogripal & 2 & 1,9 \\
Prurito & 3 & 2,8 \\
Poliartralgia & 1 & 0,9 \\
Erupción cutánea & 2 & 1,9 \\
Reacción paradójica neurológica & \\
\hline
\end{tabular}

† El caso de M. kansasii se excluyó del analisis de efectos adversos y desenlace de la terapia. 
con la recaída $(p<0,05)$. No se encontró asociación entre la recaída por tuberculosis pulmonar y el resto de las características demográficas, clínicas, epidemiológicas o microbiológicas evaluadas.

\section{Efectos secundarios asociados a la terapia antituberculosa}

La incidencia de efectos secundarios asociados al tratamiento fue de $8,5 \%$, principalmente de leve y moderada seriedad, que no requirieron interrupción del tratamiento (cuadro 4). Todos los efectos secundarios se resolvieron completamente con el manejo instaurado y no se presentó mortalidad asociada a ellos.

El caso de hipersensibilidad a pirazinamida tuvo manifestaciones cutáneas, requirió tratamiento con esteroides orales y protocolo de reintroducción de medicamentos (6), con lo cual se logró la tolerancia. Además, se presentaron dos efectos adversos graves de tipo neurológico, uno de ellos indicativo de síndrome cerebeloso y otro con características de focalización motora y sensitiva. Ambos casos se estudiaron bajo hospitalización en el tercer nivel de atención, para descartar lesiones del sistema nervioso central, trastornos metabólicos, vasculares y otras enfermedades infecciosas como causa de ellos. No se encontró otra explicación para estas reacciones y se interpretaron como idiosincrásicas a la terapia; finalmente, hubo resolución completa del cuadro neurológico.

No se encontró asociación entre la incidencia de los efectos secundarios y las características demográficas, clínicas o las enfermedades concomitantes de los pacientes al momento del diagnóstico de tuberculosis pulmonar. Sin embargo, se observó una tendencia hacia una mayor frecuencia de efectos adversos en la población mayor de 55 años (20\%).

\section{Discusión}

Las principales metas de los Programas de Control de la Tuberculosis son la detección precoz y el diagnóstico y tratamiento oportunos, con el objetivo de reducir la mortalidad y la morbilidad y de interrumpir la cadena de transmisión.

Varios estudios han denominado como "retraso diagnóstico" al tiempo comprendido entre el inicio de síntomas y el diagnóstico de tuberculosis pulmonar, y lo han reportado con un promedio de 1 a 2 meses $(7,8)$. En nuestro país, en un estudio realizado en Bucaramanga se encontró una mediana de 120 días para establecer el diagnóstico de la enfermedad, asociada a factores como el desempleo y la falta de cobertura en salud (8). En otros países han reportado otros factores que contribuyen a un mayor tiempo para establecer el diagnóstico, como son el costo de la atención médica, el desconocimiento de la enfermedad por parte del paciente, los prolongados tiempos de espera en los servicios de salud, y la falta de sospecha y búsqueda de la enfermedad por parte del personal de salud (9-15).

Tomando en consideración que, según la normatividad nacional e internacional, todo sintomático respiratorio (tos y expectoración por más de 15 días) debe estudiarse mediante baciloscopia seriada, el tiempo que toma hacer el diagnóstico de tuberculosis pulmonar a partir del inicio de síntomas no debería superar un mes calendario en condiciones ideales.

En el presente estudio, el tiempo para establecer el diagnóstico de tuberculosis pulmonar mediante baciloscopia fue mayor de 9 semanas en 52,1\% de los pacientes (cuadro 2). Sin embargo, no se exploró el tiempo entre el inicio de los síntomas y la consulta al servicio de salud, ni otros factores que pudieron haber intervenido en ello. En razón de lo anterior, es recomendable adelantar estudios orientados a identificar posibles causas del retraso diagnóstico, como el descrito en esta cohorte.

Los estudios radiográficos mostraron cavernas sólo en $33,6 \%$ de los pacientes y el hallazgo principal cuando los lóbulos superiores estuvieron afectados ( $72 \%$ de los casos) fue infiltrado reticulonodular, identificado como uno de los cambios iniciales en el desarrollo de la enfermedad. En 13 de 104 casos $(12,5 \%)$ se encontró atelectasia, consolidación o ambas, sin asociación a cavernas, hallazgos que sin estar acompañados de estudio microbiológico apropiado, podrían ser interpretados como neumonía no tuberculosa. En general, los hallazgos radiográficos fueron diversos y no correspondieron a los tradicionalmente concebidos para la enfermedad. De manera global, éstos fueron compatibles con tuberculosis posterior a la primaria, que indica reactivación de la enfermedad a partir de una infección primaria (16).

Durante el 2006, último año de inclusión de pacientes para este estudio, en Colombia se notificaron 11.128 pacientes con diagnóstico de tuberculosis y se evaluó el 53,7\% (5.978) de éstos para infección concomitante con $\mathrm{VIH}$; se encontró una prevalencia de la infección concomitante $\mathrm{VIH}$ de $6,5 \%$ en todos los casos y $3,5 \%$ en los 
casos nuevos (17), mientras que la infección concomitante tuberculosis/VIH encontrada en esta cohorte fue de $5,7 \%$, lo que supera lo descrita en casos nuevos a nivel nacional. A nivel mundial, se estima que $11 \%$ de todos los casos nuevos de tuberculosis tienen infección simultánea con $\mathrm{VIH}$, siendo éste el factor de riesgo conocido más importante para la progresión hacia enfermedad activa por tuberculosis en las personas con infección latente.

Con frecuencia, la tuberculosis es la primera manifestación de la enfermedad por VIH, como se vio representado en los casos de este estudio, y es la infección oportunista más común en estos pacientes (18). La prueba para VIH debe ofrecerse de rutina, mediante un consentimiento informado, a todos los pacientes con diagnóstico de tuberculosis según guías nacionales e internacionales (19). Establecer la tamización para VIH en todos los pacientes con diagnóstico de tuberculosis, tal como se realiza en programas de control prenatal en Colombia, sería una estrategia de gran impacto para los Programas de Control de la Tuberculosis.

Los estudios de resistencia a medicamentos antituberculosos realizados en Colombia en 1992, 1999-2000 y 2004-2005 (20), por el Instituto Nacional de Salud, mostraron que la tasa de resistencia inicial a los medicamentos de primera línea en el país es de 2,38\%. Los hallazgos de resistencia en este trabajo son similares a los datos del último estudio nacional. No obstante, se ha reportado una proporción superior de resistencia primaria a los medicamentos de primera línea en el municipio de Buenaventura $(21,22)$, con una población atendida frecuentemente en la ciudad de Cali, lo cual se convierte en un factor epidemiológico que se debe tener en cuenta en el abordaje de los casos.

Durante el presente estudio se hizo evidente la importancia de disponer de un protocolo claro para el manejo de la tuberculosis resistente a los medicamentos de primera línea y de personal entrenado para ello. El pobre o nulo acceso a los medicamentos antituberculosos de segunda línea fue una situación enfrentada por los pacientes con este perfil de resistencia, razón por la cual se ha recomendado fortalecer la estrategia DOTS, con disponibilidad adecuada de medicamentos de segunda línea, en las áreas donde se ha documentado resistencia (23). Cabe resaltar que el único caso que fracasó a la terapia antituberculosa en esta cohorte, estaba asociado con resistencia a isoniacida y estreptomicina, e hipersensibilidad a la pirazinamida.

La proporción de casos curados al final de la terapia fue de $87,6 \%$, lo cual supera la cifra del último reporte global de tuberculosis de la OMS, el cual mostró una tasa de curación en la región de las Américas de 78\% para el año 2006 (17). En el mismo reporte, Colombia mostró una tasa de éxito (curación y tratamiento completo) de $71 \%$ y una cobertura de la estrategia DOTS de $60 \%$. El anterior hallazgo fortalece la idea de que la meta de curación de $85 \%$, propuesta por la OMS, es posible en el contexto de la estrategia "Alto a la TB". Esto se ha evidenciado en otras cohortes que han registrado el aumento en las tasas de éxito terapéutico desde la implementación de la estrategia DOTS $(24,25)$.

En nuestra cohorte, 6 de los 9 pacientes que abandonaron la terapia lo hicieron por causas asociadas a la violencia (delincuencia común) y detención en prisión (figura 1).

En estudios previos de cohortes de pacientes tratados bajo la estrategia DOTS en Colombia, se han encontrando como factores de riesgo para el abandono: la falta de información al paciente y la familia sobre la enfermedad, el retraso diagnóstico mayor de 2 meses, ser portador del VIH y haber estado detenido (26). En el presente estudio, no se identifico este último previo al ingreso, pero una vez en esta condición, no se logró completar el seguimiento satisfactoriamente debido a la comunicación restringida por parte del sistema carcelario hacia el Programa de Control de la Tuberculosis y personal clínico del estudio. A la luz de esta experiencia, sería relevante explorar la violencia como posible factor de riesgo para el abandono de la terapia antituberculosa. El porcentaje de abandono encontrado en este trabajo $(8,5 \%)$ representó la mitad de lo reportado para el año 2006 en Cali (3).

Es posible que las actividades de acompañamiento cercano por un equipo interdisciplinario dedicado exclusivamente a los pacientes durante el tratamiento y el seguimiento, los incentivos de transporte y alimentación, y el hecho de haber recibido medicamentos antituberculosos solamente de administración oral, sean condiciones que hayan favorecido el éxito terapéutico en esta cohorte. Por lo anterior, consideramos que podría ser de gran utilidad que los Programas de Control de la Tuberculosis en la región implementaran las 
estrategias mencionadas, para reducir potencialmente el porcentaje de abandono.

En algunas revisiones sistemáticas se ha evidenciado que los programas de control de la tuberculosis en diferentes regiones del mundo se valen de estrategias complementarias al DOTS, para lograr resultados de éxito y cumplimiento; entre ellas se incluyen llamadas y visitas recordatorias para los pacientes con faltas en el tratamiento, incentivos económicos, de alimentación y transporte, y abordajes terapéuticos ajustados a las necesidades y preferencias del paciente (27).

Los efectos secundarios a la medicación y su incidencia en esta población se encontraron dentro de lo esperado para la terapia administrada a los pacientes (28).

Nuestros hallazgos tienen la limitación de provenir de una población de bajo riesgo para el desarrollo de efectos secundarios a los medicamentos antituberculosos. Sin embargo, no existen publicaciones previas en nuestro país que describan los detalles de nuestras observaciones y el seguimiento de cohortes en los programas no informa sobre la presentación dichos efectos. El adecuado manejo de los efectos secundarios es parte de los elementos más importantes de la estrategia "Alto a la TB", para lograr resultados de éxito terapéutico y evitar casos de abandono por intolerancia a la medicación.

Finalmente, los hallazgos y observaciones descritas son una herramienta que puede ser utilizada por los Programas de Control de la Tuberculosis para el fortalecimiento de la estrategia propuesta por la OMS y evidencian información básica que debe ser recordada por los trabajadores de salud que se enfrentan al diagnóstico y manejo de la tuberculosis. La investigación de las causas del retraso diagnóstico de la tuberculosis, la implementación de medidas que permitan la captación temprana de casos nuevos y su oportuno tratamiento, son fundamentales para disminuir la transmisión de la tuberculosis en la comunidad. Asimismo, se requiere estudiar e intervenir las causas de abandono del tratamiento, con el propósito de disminuir la aparición de tuberculosis resistente y las complicaciones de la enfermedad. La disponibilidad de medicamentos antituberculosos de segunda línea, el entrenamiento para el manejo de casos de tuberculosis resistente a los medicamentos de primera línea y el adecuado manejo de los efectos adversos, son parte de los elementos más importantes de la estrategia DOTS para lograr buenos resultados de éxito terapéutico y evitar el aumento en el número de casos de pacientes con tuberculosis resistente.

La tuberculosis es una enfermedad prevenible y curable: es primordial integrar esfuerzos nacionales para lograrlo.

\section{Agradecimientos}

A Judith Bastidas, auxiliar de enfermería participante en el proyecto, por su gran dedicación al seguimiento y atención de los pacientes.

A Antonio José Triana y al resto del personal del Programa de Control de la Tuberculosis de la Secretaria de Salud Pública Municipal de Cali y de las Instituciones de Salud participantes.

\section{Conflicto de interés}

Los autores no tenemos conflictos de intereses.

\section{Financiación}

Estudio cofinanciado por la Unión Internacional contra la Tuberculosis y Enfermedades Respiratorias, el Centro Colombiano de Investigación en Tuberculosis (CCITB), CIDEIM y Colciencias (Contrato 431 de 2004, Código 34261817270).

\section{Referencias}

1. World Health Organization. Global tuberculosis controlepidemiology, strategy, financing. WHO Report 2009 (WHO/ HTM/TB/2009.411). Geneva: World Health Organization; 2009.

2. World Health Organization. The Stop TB Strategy: Building on and enhancing DOTS to meet the TB-related Millennium Development Goals. Geneva: World Health Organization; 2008.

3. Secretaría de Salud Pública Municipal de Cali. Boletín Epidemiológico. Santiago de Cali: Secretaría de Salud Pública Municipal de Cali; 2007.

4. Rieder H, van Deun A, Kam KM, Kim SJ, Chonde TM, Trébucq A, et al. Priorities for tuberculosis bacteriology services in low-income countries. Second edition. Paris: International Union Against Tuberculosis and Lung Disease; 2007.

5. Kent P, Kubica G. Public health mycobacteriology: a guide for the level III laboratory. Atlanta: U.S. Department of Health and Human Services, Public Health Service, Centers for Disease Control; 1985.

6. Caminero JA. Tuberculosis guide for specialist physicians. Paris: International Union Against Tuberculosis and Lung Disease; 2004.

7. Pirkis JE, Speed BR, Yung AP, Dunt DR, Maclntyre CR, Plant AJ. Time to initiation of anti-tuberculosis treatment. Tuber Lung Dis. 1996;77:389-90.

8. Cáceres-Manrique F, Orozco-Vargas LC. Demora en el diagnóstico de tuberculosis pulmonar en una región de Colombia. Rev Salud Pública. 2008;10:94-104. 
9. Rajeswari $R$, Chandrasekaran V, Suhadev $M$, Sivasubramaniam S, Sudha G, Renu G. Factors associated with patient and health system delays in the diagnosis of tuberculosis in South India. Int J Tuberc Lung Dis. 2002;6:789-95.

10. Godfrey-Faussett P, Kaunda H, Kamanga J, van Beers S, van Cleeff M, Kumwenda-Phiri R, et al. Why do patients with a cough delay seeking care at Lusaka urban health centres? A health systems research approach. Int J Tuberc Lung Dis. 2002;6:796-805.

11. Lönnroth K, Thuong LM, Linh PD, Diwan VK. Delay and discontinuity -a survey of TB patients' search of a diagnosis in a diversified health care system. Int J Tuberc Lung Dis. 1999;3:992-1000.

12. Salaniponi FM, Harries AD, Banda HT, Kang'ombe C, Mphasa N, Mwale A, et al. Care seeking behaviour and diagnostic processes in patients with smear-positive pulmonary tuberculosis in Malawi. Int $\mathrm{J}$ Tuberc Lung Dis. 2000;4:327-32.

13. Sarmiento K, Hirsch-Moverman Y, Colson PW, El-Sadr W. Help-seeking behavior of marginalized groups: a study of TB patients in Harlem, New York. Int J Tuberc Lung Dis. 2006;10:1140-5.

14. Selvam JM, Wares F, Perumal M, Gopi PG, Sudha G, Chandrasekaran V, et al. Health-seeking behaviour of new smear-positive TB patients under a DOTS programme in Tamil Nadu, India, 2003. Int J Tuberc Lung Dis. 2007;11:161-7.

15. Ward J, Siskind V, Konstantinos A. Patient and health care system delays in Queensland tuberculosis patients, 1985-1998. Int J Tuberc Lung Dis. 2001;5:1021-7.

16. Daley CK, Gotway MB, Jasmer RB. Radiographic manifestations of tuberculosis: A primer for clinicians. Second edition. San Francisco: Francis J. Curry National Tuberculosis Center; 2006. p. 2-23.

17. World Health Organization. Global Tuberculosis Control, Surveillance, Planning, Financing. WHO Report 2008 (WHO/ HTM/TB/2008.393): Geneva: World Health Organization; 2008.

18. Williams G. Best practice for the care of patients with tuberculosis. A guide for low-income countries. Paris:
International Union Against Tuberculosis and Lung Disease; 2007.

19. World Health Organization. Treatment of tuberculosis: Guidelines for national programmes. Third edition. Geneva: World Health Organization; 2003.

20. Garzón MC, Angée DY, Llerena C, Orjuela DL, Victoria JE. Vigilancia de la resistencia del Mycobacterium tuberculosis a los fármacos antituberculosos, Colombia 2004-2005. Biomédica. 2008;28:319-26.

21. Laserson KF, Osorio L, Sheppard JD, Hernández H, Benítez AM, Brim S, et al. Clinical and programmatic mismanagement rather than community outbreak as the cause of chronic, drug-resistant tuberculosis in Buenaventura, Colombia, 1998. Int J Lung Dis. 2000:4:67383.

22. Moreira CA, Hernández HL, Arias NL, Castaño MC, Ferro BE, Jaramillo E. Resistencia inicial a drogas antituberculosas en Buenaventura, Colombia. Biomédica. 2004;24(Suppl.1):73-9.

23. Farmer $\mathbf{P}$, Kim JY. Community based approaches to the control of multidrug resistant tuberculosis: introducing "DOTS-plus". BMJ. 1998;317:671-4

24. Bao QS, Du YH, Lu CY. Treatment outcome of new pulmonary tuberculosis in Guangzhou, China 19932002: a register-based cohort study. BMC Public Health. 2007;7:344.

25. Thomas A, Gopi PG, Santha T, Chandrasekaran V, Subramani R, Selvakumar N, et al. Predictors of relapse among pulmonary tuberculosis patients treated in a DOTS programme in South India. Int J Tuberc Lung Dis. 2005;9:556-61.

26. Cáceres F, Orozco LC. Incidencia y factores asociados al abandono del tratamiento antituberculoso. Biomédica. 2007;27:498-504.

27. Maher D, Gupta R, Uplekar M, Dye C, Raviglione M. Directly observed therapy and treatment adherence. Lancet. 2000;356:1031-2.

28. Blomberg B, Spinaci S, Fourie B, Laing R. The rationale for recommending fixed-dose combination tablets for treatment of tuberculosis. Bull World Health Organ. 2001;79:61-8. 